\title{
Improved Reliability of FPGA-Based PUF Identification Generator Design
}

CHONGYAN GU, NEIL HANLEY, and MÁIRE O’NEILL, CSIT, ECIT, Queen's University Belfast, Queen's Road, United Kingdom, BT3 9DT

\begin{abstract}
Physical unclonable functions (PUFs), a form of physical security primitive, enable digital identifiers to be extracted from devices, such as field programmable gate arrays (FPGAs). Many PUF implementations have been proposed to generate these unique $n$-bit binary strings. However, they often offer insufficient uniqueness and reliability when implemented on FPGAs and can consume excessive resources. To address these problems, in this article we present an efficient, lightweight, and scalable PUF identification (ID) generator circuit that offers a compact design with good uniqueness and reliability properties and is specifically designed for FPGAs. A novel post-characterisation methodology is also proposed that improves the reliability of a PUF without the need for any additional hardware resources. Moreover, the proposed post-characterisation method can be generally used for any FPGA-based PUF designs. The PUF ID generator consumes $8.95 \%$ of the hardware resources of a low-cost Xilinx Spartan-6 LX9 FPGA and 0.81\% of a Xilinx Artix-7 FPGA. Experimental results show good uniqueness, reliability, and uniformity with no occurrence of bit-aliasing. In particular, the reliability of the PUF is close to $100 \%$ over an environmental temperature range of $25^{\circ} \mathrm{C}$ to $70^{\circ} \mathrm{C}$ with $\pm 10 \%$ variation in the supply voltage.
\end{abstract}

CCS Concepts: • Security and privacy $\rightarrow$ Tamper-proof and tamper-resistant designs; Hardwarebased security protocols; Embedded systems security;

Additional Key Words and Phrases: Physical unclonable functions (PUFs), identification generation, authentication, field programmable gate arrays (FPGAs), reliability

\section{ACM Reference Format:}

Chongyan Gu, Neil Hanley, and Máire O’Neill. 2017. Improved reliability of FPGA-based PUF identification generator design. ACM Trans. Reconfigurable Technol. Syst. 10, 3, Article 20 (May 2017), 23 pages.

DOI: http://dx.doi.org/10.1145/3053681

\section{INTRODUCTION}

Physical unclonable functions (PUFs) allow hardware devices to be physically and uniquely identified by associating unique $n$-bit binary string identifiers with the devices, such as field-programmable gate arrays (FPGAs). This has opened the door to a number of new security-orientated FPGA design opportunities, such as intellectual property protection, cloning prevention, or complex security-on-chip designs. PUF designs have been extensively studied for hardware targets, both application specific ICs (ASICs) and FPGAs, and recently have begun to appear in commercial products such as the latest version of the NXP SmartMX micro-controller integrated circuit (IC) targeted at transport and banking markets [Intrisic-ID 2015] and the new Microsemi SmartFusion2 SoC FPGA line [Microsemi 2015]. The Xilinx UltraScale+ devices also have

This work is supported by EPSRC (EP/N508664/-CSIT2), by the KeyHAS project, the R\&D program of IITP/MSIP (Study on secure key hiding technology for IoT devices), and by the SPARKS project, funded by EU 7th Framework Programme (FP7/2007-2013, grant agreement no. 608224; www.project-sparks.eu. Authors' addresses: C. Gu, N. Hanley, and M. O'Neill, Centre for Secure Information Technologies (CSIT), Institute of Electronics, Communications \& Information Technology (ECIT), Queen's University Belfast; emails: \{cgu01, n.hanley\}@qub.ac.uk,m.oneill@ecit.qub.ac.uk.

Permission to make digital or hard copies of all or part of this work for personal or classroom use is granted without fee provided that copies are not made or distributed for profit or commercial advantage and that copies bear this notice and the full citation on the first page. Copyrights for components of this work owned by others than the author(s) must be honored. Abstracting with credit is permitted. To copy otherwise, or republish, to post on servers or to redistribute to lists, requires prior specific permission and/or a fee. Request permissions from Permissions@acm.org.

(C) 2017 ACM 1936-7406/2017/05-ART20 $\$ 15.00$

DOI: http://dx.doi.org/10.1145/3053681 
the option of using PUF for masking of the key used for bitstream encryption (note that it is not accessible for user designs) and have published white papers on how to enhance boot security with the use of a user designed "soft" PUF in the FPGA fabric [Peterson 2015]. Ideally, PUFs should offer a tamper-evident, unpredictable, and unclonable solution. However, some PUF designs can be predicted by using machinelearning attacks [Rührmair et al. 2010] and physical attacks, for example, fault injection [Delvaux and Verbauwhede 2014], side channel attacks [Mahmoud et al. 2013]. Recently, countermeasures have also been proposed to improve the physical security of PUF designs, for example, modeling attack resistant PUFs [Kumar and Burleson 2014; Vijayakumar and Kundu 2015]. Some of these proposals will be discussed in the next section. One advantage of using PUF over other approaches is that any device tampering can affect the PUF response and hence might be detected. Instead of storing a preset identifier in non-volatile memory (NVM) they exploit process variation effects via a "variability aware" circuit to generate a unique $n$-bit binary string identifier (response) from an FPGA when given a corresponding $N$-bit input (challenge). Since, ideally, each $i$ th challenge, $C_{i}$, uniquely maps to exactly one response, $R_{i}$, without noise, they can be grouped together into so-called challenge-response pairs (CRPs), where $C R P_{i}=\left\{C_{i}, R_{i}\right\}$.

To enable practical use of the identifiers generated using the FPGA-based PUFs, they have to (1) be unique so no two devices map to the same ID, (2) offer high reliability to ensure that a device can repeatedly return the correct ID with as few noisy bits as possible, and (3) be efficient and feasible to implement on an FPGA. However, to date widespread adoption in FPGAs has been limited as PUFs are difficult to implement and integrate on such devices, can require considerable FPGA logic resources, and have insufficient tolerance to temperature and voltage variations.

Uniqueness in PUF IDs is inherently difficult to achieve as they exploit manufacturing process variations, which FPGA microelectronic designers strive to minimize. If the level of variability is not sufficient, then two challenges, $C_{1}$ and $C_{2}$, may map to the same or related responses for different FPGA instances, that is, $C_{1} \rightarrow R_{1}$ and $C_{2} \rightarrow R_{1}$. Furthermore, as FPGAs have highly regular and scalable architectures to allow them to implement arbitrary logic functions efficiently, this directly affects the implementation options. The design tools create layouts with unbalanced routing and interconnects with large capacitance, both of which introduce bit biases and skew in PUF response bits, that is, bit-aliasing.

Reliability in PUF ID generator designs is affected by environmental variations, the most significant of which are core supply voltage and temperature fluctuations. If an FPGA's core supply voltage levels diverge significantly from the recommended value gate, then delays will change and can cause incorrect ID responses. Similarly, elevated local temperatures will impact an FPGA's performance and response bit accuracy. Changes in temperature cause transistor threshold voltages to decrease and carrier mobility to increase: The former tends to speed up a circuit, while the latter tends to slow it down. Depending on which effect is dominant, a circuit may show either negative temperature dependence if the delay increases with temperature, positive temperature dependence if it decreases with temperature, or mixed temperature dependence if the trend is non-uniform [Wolpert and Ampadu 2012]. Ultimately, both types of environmental variation causes reliability issues.

In previous work by the authors [Gu et al. 2014], a novel FPGA-based PUF ID generator was proposed that consumes minimal FPGA logic resources and can be easily scaled to form an $n$-bit PUF ID generator and implemented in a low-cost FPGA device, such as a Spartan-6 LX9. Experimental results demonstrate that this PUF ID generator design achieves good uniqueness and reliability. Each 1-bit ID cell is implemented as a hard-macro on an FPGA ensuring balanced and stable routing for reliable operation. 
This is important when generating identifiers under different environmental conditions and minimizes statistical bias. Although the reliability of our previous work (93\%) is sufficient for ID generation and authentication, for other applications, such as key generation, a more robust response is required. Ideally the aim is to improve the reliability result without utilising extra hardware resource on an FPGA, which is one focus of this article.

In this article, we build on our previous work and propose a reliable characterisation process. We also provide a more complete analysis of the FPGA-based PUF ID generator. Specifically, our scientific research contributions are as follows:

-We propose a reliable and efficient post-processing characterisation process, which can be implemented on FPGA without any additional hardware resources. This characterisation process can be utilized to improve the reliability of any FPGA-based PUF ID generator design. It is employed to enhance the reliability of the 128-bit PUF ID generator previously proposed by the authors [Gu et al. 2014].

- The application of this automated characterisation process is presented, and an improvement in the reliability of the 128-bit PUF ID generator from $93.93 \%$ to $98.74 \%$ without the requirement of any additional hardware resources, and to $99.60 \%$ when simple temporal majority voting post-processing is also employed, is shown.

-A more comprehensive evaluation of the PUF ID generator design previously proposed by the authors [Gu et al. 2014] is also presented and includes an analysis of uniformity and bit-aliasing, with results of $51.06 \%$ and $56.48 \%$ achieved, respectively, pre-characterisation.

- The proposed improved 128-bit PUF ID generator is shown to achieve good overall results in terms of uniqueness, uniformity, and bit-aliasing, with values of $45.60 \%$, $50.60 \%$, and $56.48 \%$, respectively, using the proposed characterisation process and a further improvement to $45.60 \%, 50.54 \%$ and $56.58 \%$, respectively, using temporal majority voting.

The rest of this article is organized as follows. Section 2 discusses the related work of PUF designs. Section 3 introduces the principle of the PUF ID generator's operation. The implementation of the 128-bit PUF ID generator design is described in Section 4 and the post-characterisation process is outlined in Section 5. The evaluation of the proposed improved PUF ID generator design is given in Section 6 to validate the work. Finally, conclusions are drawn in Section 7.

\section{RELATED WORK}

Since the first concrete implementation of a PUF was proposed [Pappu et al. 2002], many researchers have reported a range of different PUFs targeting both ASICs and FPGAs, for example, static RAM (SRAM) PUFs [Guajardo et al. 2007; Holcomb et al. 2009], Latch PUF [Su et al. 2008], Flip-flop PUF [Maes et al. 2008], Buskeeper PUF [Simons et al. 2012], Butterfly PUF [Kumar et al. 2008], Ring Oscillator (RO) PUF [Suh and Devadas 2007; Murphy et al. 2012], Configurable RO (CRO) PUF [Yu et al. 2012], Arbiter PUF [Gassend et al. 2002; Gu et al. 2016], Bistable Ring (BR) PUF [Chen et al. 2011], processor-based PUF [Maiti and Schaumont 2012], and reconfigurable PUF (rPUF) [Kursawe et al. 2009]. Also, Charles et al. [Herder et al. 2014] and Sklavos [2013] provide a detailed introduction to PUF-based security analysis and implementation. The RO PUF designs exploit the difference in period between two identical ring oscillators by incrementing two counters and then comparing the value reached in a given timeframe. This structure at a minimum requires two configurable logic blocks (CLBs) on FPGA, even though strategies exist to re-use oscillators [Maiti et al. 2012]. The Arbiter PUF uses $n$-bit differential delay lines and a latch arbiter to generate a 1-bit PUF response. It is difficult to implement this design on FPGA as it requires the 
whole structure to be balanced to generate 1-bit and then duplicated $k$ times. Although Majzoobi et al. [2014] and Hori et al. [2014] implemented Arbiter PUFs on FPGAs, they introduced an extra tuning circuit or reported results with low uniqueness. In memory-based PUFs, like SRAM PUFs, the initial state of static RAM cells, formed by two cross-coupled inverters (also often known as a bistable latch) in FPGAs, is exploited to produce IDs based on different memory blocks on different FPGAs. However, SRAM PUFs require a device power-up operation to generate each ID. Although most FPGAs have SRAM memory, some SRAMs have an initial state that prevents them entering a random value during the start up stage. To address this, Kumar et al. [2008] proposed a logical alternative called Butterfly PUF to emulate the behavior of an SRAM PUF on Virtex-5 FPGAs. It can be invoked at any time rather than only at power-up. It operates using two cross-coupled latches forming a bistable circuit, where the preset/clear force it into metastability. It still suffers from issues due to metastability, and indeed not all FPGAs feature preset/reset pins in the required format. They reported $94 \%$ reliability over temperature variations; however, reliability over voltage changes is not provided. For 64 Butterfly PUF cells, 130 slices are consumed.

Improving the reliability has been the subject of much research with both SRAM and RO PUF designs. Efforts to improve the reliability of SRAM PUF have been proposed by many researchers, for example, Bhargava and Mai [2014], Gary and Kim [2014] and Cortez et al. [2013]. However, aging testing-based reinforcement techniques or special circuitry are required. Guajardo et al. [2007] and Bohm et al. [2011] utilise error correction codes, $\mathrm{BCH}$ code or repetition code, to reduce the error rate for SRAM PUFs. A fuzzy extractor is used for error correction to enhance the reliability in the SRAM PUF design proposed by Holcomb et al. [2013], the Flip-flop PUF design by Maes et al. [2008], and the Butterfly PUF design by Kumar et al. [2008]. All these post processing methods incur additional hardware resource usage. Temporal majority voting (or 1-out-of- $k$-method) is a straightforward and simple way to reduce noise, for example, employed by Bolotnyy and Robins [2007]. However, its usefulness depends on the level of noise. For example, in the case of $50 \%$ noise, even if temporal majority voting is applied, the result cannot be improved. Hence, a lightweight reliability improvement is needed to enhance the reliability of FPGA-based PUF designs in general.

To distinguish between different intrinsic PUF designs, Guajardo et al. [2007] introduced two PUF subtypes with regard to the behavior of CRPs, namely "Weak PUF" and "Strong PUF." Weak PUFs exhibit the following characteristics: (1) they may have very few challenges, and in the extreme case they may generate just one response; and (2) it is assumed that an attacker cannot access the response of Weak PUFs as one or a few CRPs could be used to build a model of the security system. The previously mentioned SRAM PUF and Butterfly PUF are examples of Weak PUFs. For many applications, their responses can be useful for key generation as an intrinsic key in place of secure memory. Compared to other key storage approaches in which keys are stored in NVM, the key is intrinsically linked to the physical hardware of the device itself. Furthermore, Weak PUFs are low cost, since they do not need any special manufacturing process. Compared to Weak PUFs, Strong PUFs have the following characteristics: (1) They may have many possible CRPs and (2) an attacker may have access to the CRPs; however, it should be impossible for them to determine or attack the CRPs in a given timeframe, for example, a few days or weeks.

Practical realisations of the Strong PUF definition of Guajardo et al. [2007] has proven to be somewhat more difficult than originally anticipated. For example, the Arbiter PUF was an example of a Strong PUF; however, it is known that Arbiter PUFs can be modeled as linear additive models [Rührmair et al. 2010], and recent work has shown how to attack the non-linearity of XOR-Arbiter PUFs using reliability-based evolution strategies [Becker 2015]. Hence, in more recent literature, a Strong PUF is 


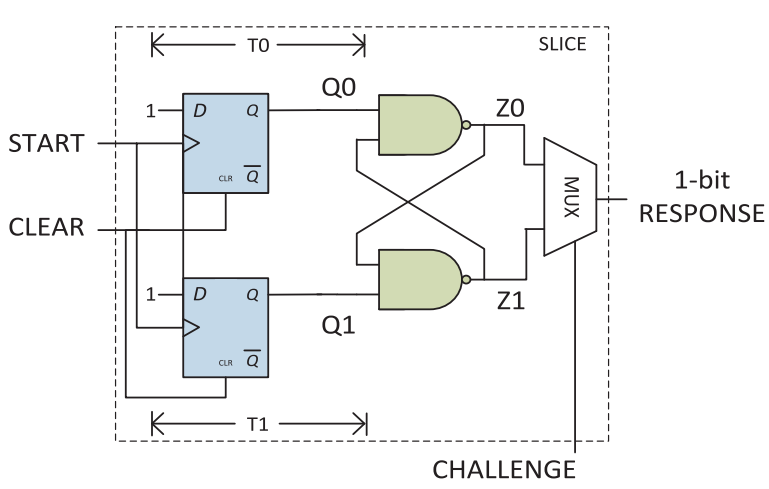

Fig. 1. circuit design.

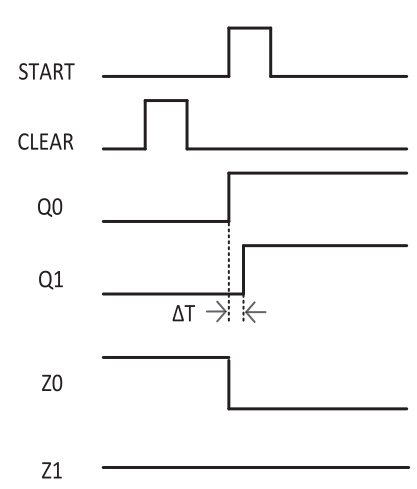

Fig. 2. Timing diagram.

required to have a number of CRPs that scales exponentially with the circuit area, but no other constraints such as resistance against modeling are imposed. For Weak PUF, such attacks do not apply, as it is assumed that there is no external access to the response for an attacker. Hence, machine-learning attacks are not considered here. For Strong PUFs, poor reliability is one reason why XOR PUFs can be attacked, as described in Becker [2015]. Hence, the characterisation process presented in this article to help achieve high reliability may be helpful for Strong PUFs to protect against machine-learning attacks.

\section{PUF ID GENERATOR CIRCUIT DESIGN}

The previously proposed PUF ID generator design by the authors [Gu et al. 2014] comprises of $n$ elementary 1-bit PUF ID cells and is designed to fit compactly in one FPGA slice, as shown in Figure 1. An $n$-bit PUF ID generator circuit is formed by instantiating an array of $n$ 1-bit ID cells. A 1-bit response is generated as follows: two matched time delay paths, $T O$ and $T 1$, implemented by two D type flip-flops are excited simultaneously by the rising edge of a START signal connected to their clock pins after first being reset by $C L E A R$; since flip-flops are coarse-grained delay components, the rising edge on their $Q$ outputs propagate the excited signal differently, thus racing against each other; the timing of two delay lines will differ due to underlying manufacturing variability; cross-coupled NAND gates are utilized to decide which transition arrived first and sets their output to either binary 10 or 01 . A timing diagram of the 1-bit PUF ID generator design is shown in Figure 2. It can be seen that a CLEAR signal is first activated to reset the circuit, and on the rising edge of a START signal the delay paths are activated. The output signals, $Z 0$ and $Z 1$, will be 01 when $Q 0$ and $Q 1$ are 10 whenever the arrival time of the delay path $T O$ is faster. A multiplexer outputs a unique 1-bit response depending on the value of the challenge. This PUF ID generator bit generation circuitry requires two LUTs, two flip-flops and one multiplexer per bit.

The use of cross-coupled NAND gates as an arbiter ensures that the feedback paths are balanced and symmetrical and contribute minimally to $T 0$ and $T 1$. The arbiter design also increases reliability as the effects on each feedback path are equally balanced. Previous work by Lim et al. [2005] uses a D-latch for the arbiter, but it introduces a $10 \%$ skew on the response.

To maximize variation and to avoid bit aliasing in the ID responses, the wiring paths must be placed and routed as symmetrically as possible to minimize the nominal delay difference between the two paths. In FPGAs, this can be accomplished by manual routing and timing analysis, but due to the natural architecture of FPGAs this is inherently 


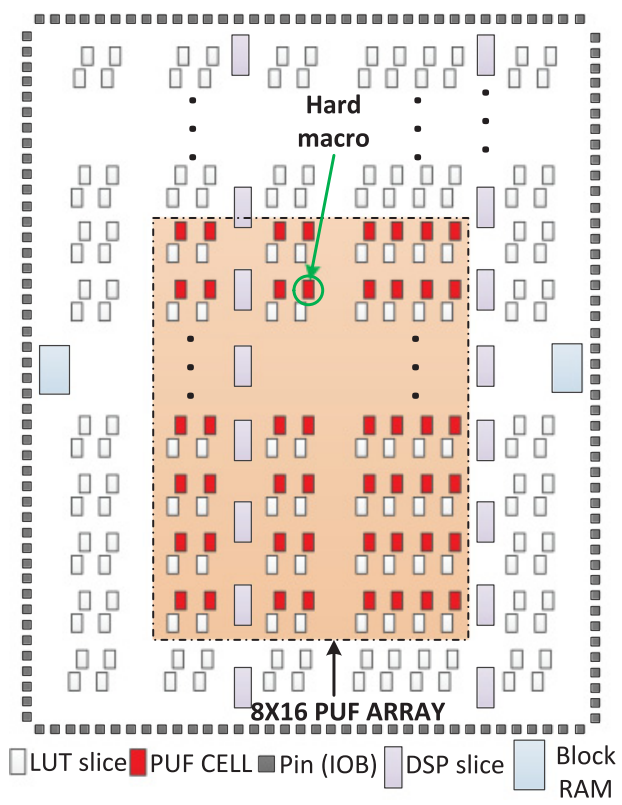

Fig. 3. Floor plan of a 128-bit PUF ID generator based on a 1-bit single slice hard macro.

problematic. Careful place and route in the target device ensures an estimated delay difference between the paths of only 10ps according to the design tool.

Due to external influences as mentioned previously (temperature and supply voltage), some bits will be unstable and vary between 0 and 1 . The straightforward solution to this problem is to obtain each response bit $N(N=5$ for this work) times and then use the majority as the correct bit. As the number of repetitions increases, the probability of an undecipherable error decreases proportionately. This does not work, however, when significant instability in the bit response occurs. In the next section, a characterisation process is proposed to address instability and improve reliability.

\section{IMPLEMENTATION OF PROPOSED PUF ID GENERATOR DESIGN}

The previously proposed $n$-bit PUF ID generator design is implemented in Xilinx Spartan-6 FPGA and each bit is implemented as a hard macro, as shown in Figure 3. The floor plan location is set by declaring location (LOC) constraints using Xilinx's Unified Constraints Format (UCF) file.

To ensure that the ID response bits are a function of device variability only, it is essential that all circuit elements are identical and routing is balanced. Otherwise, the response bits will exhibit bit-bias due to the interconnect and layout mismatch. The authors in Suh and Devadas [2007] suggest using FPGA hard-macros as a solution to help meet strict design parameters. Therefore, in this research, the circuit elements are manually placed and routed and a hard-macro, as shown in Figure 4, is used to implement a 1-bit ID cell, which occupies exactly one slice of the target Xilinx Spartan6 FPGA device. Figure 5 shows an example of the unbalanced routing that results from automated place and route of the design, which will lead to a bias of the response. Other FPGA families can be targeted using the 1-bit ID cell by re-creating the hard-macro. A 5-bit temporal majority voting circuit for each 1-bit ID cell is also implemented as a hard macro in one slice. 


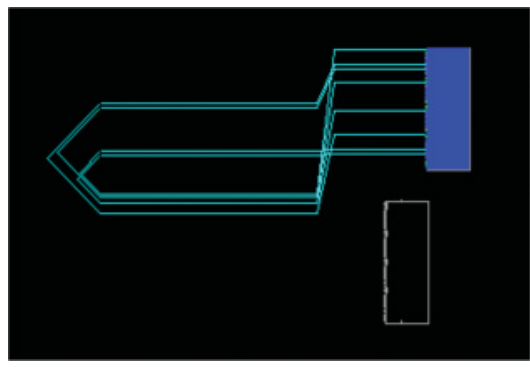

Fig. 4. Balanced routing.

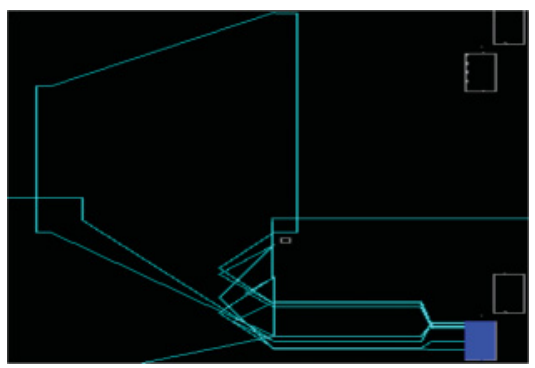

Fig. 5. Unbalanced routing.

The cross-coupled NAND gate arbiter is implemented in two LUTs, the D type flipflops are implemented in two registers, and the 2:1 selector is implemented in a multiplexer. These are easily instantiated in hardware description language (HDL) (e.g., Verilog) by using a stub file, which can be declared multiple times as desired to build $n$-bit PUF ID generators. In this work 128 hard-macros, arranged in an $8 \times 16$ array, are used to construct the 128-bit PUF ID generator as shown in Figure 3. The Xilinx Spartan-6 device employed in this work is the XC6SLX9, which has 1,430 slices, half of which are SLICEXs, a quarter of which are SLICELs and a quarter SLICEMs [Xilinx 2011]. Compared to SLICEX, the SLICEL and SLICEM primitives have wide MUXs and carry chain components. As the proposed design does not need wide MUXs or carry chains, it can be implemented in any type of slice, and for this work it is implemented in SLICEX primitives.

Since a 1-bit ID cell only occupies half a Spartan-6 slice, the remaining resources can be used to implement other functionality or alternatively a second 1-bit ID cell yielding 2-bits of an ID response per slice [Gu and O'Neill 2015]. For this work, a single bit per slice is implemented such that each ID cell occupies the upper half of the slice and the upper slice within a CLB. Utilizing only half a slice allows a great amount of flexibility in the design of complex systems, as the ID hard-macro cells can be placed anywhere on the FPGA floor plan to maximize overall resource consumption and to minimize routing congestion.

\section{POST-CHARACTERISATION METHODOLOGY}

To improve the reliability of the PUF ID generator design, a post-characterisation phase to analyse the robustness is introduced to find the unstable bits in an FPGA layout. Robustness represents how reliable the PUF ID generator design is at nominal supply voltage and room temperature. It is generally calculated by the intra-chip hamming distance between $S$ sample responses from the same PUF device under the same operational conditions. A manual characterisation process is employed to prove the feasibility of this methodology.

\subsection{Manual Characterisation Process}

A flow chart outlining the steps involved in the characterisation process is shown in Figure 7. The steps are as follows:

-Implement an $N$-bit PUF ID generator in the target FPGA, for example, Spartan-6, as shown in Figure 3.

- Generate $S$ responses from the $N$-bit PUF ID generator, where $S$ is the sample number

-Evaluate the robustness of $S N$-bit PUF ID generator responses (robustness represents the reliability of the PUF design under normal operating conditions); 


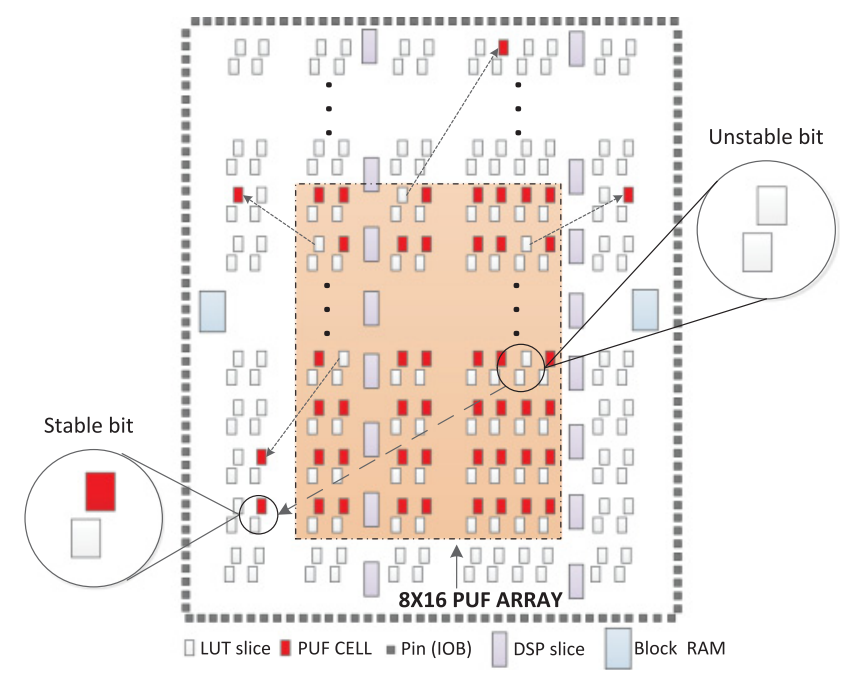

Fig. 6. Characterising the floor plan of a 128-bit PUF ID generator based on a 1-bit single slice hard macro.

-Identify the unstable bits $(m)$ and their positions in the floor plan of the FPGA,

-Find $m$ stable bits and their position as shown in Figure 6;

-Move the PUF cells in unstable bit positions to stable bit positions (repeat several times until all PUF cells positions are stable). The placement of the PUF ID cells is achieved by manually declaring LOC constraints using Xilinx's UCF in the Xilinx ISE tool;

-Update and save the final bit file with the new bit positions as the default floor plan;

- Generate the $N$-bit PUF ID generator response.

In this work the characterisation process was first manually executed following the above steps, which demonstrates the feasibility of the technique. However, an automated post-processing characterisation process was also considered to improve the efficiency of the approach.

\subsection{Automated Characterisation Process}

The flow chart for the automated characterisation process is similar to that of the manual process and is shown in Figure 8. Algorithm 1 describes in detail the execution of each step in the process and includes six phases. Similar to the manual characterisation process, the automated process explores the most stable bit output for the response to improve the reliability of the PUF ID generator design. Moreover, the automated characterisation process simplifies the post-processing and only needs to be carried out once to find out all the unstable and stable bit positions over the whole FPGA device. The execution time depends on the size of the FPGA, where the larger the FPGA, the longer the time it takes to identify the unstable and stable bit positions.

\section{EVALUATION OF PROPOSED IMPROVED PUF ID GENERATOR DESIGN}

\subsection{Experimental Setup}

The Xilinx ISE Design Suite 14.7 tool was used for the proposed design and Matlab was utilised to communicate with and test the PUF IP core, with a simple interface written to send and receive data over the USB-UART port of target FPGA boards. To evaluate the manual characterisation process, the 128-bit PUF ID generator design was implemented on a Spartan XC6SLX9 microboard, which comprises of a low-cost 


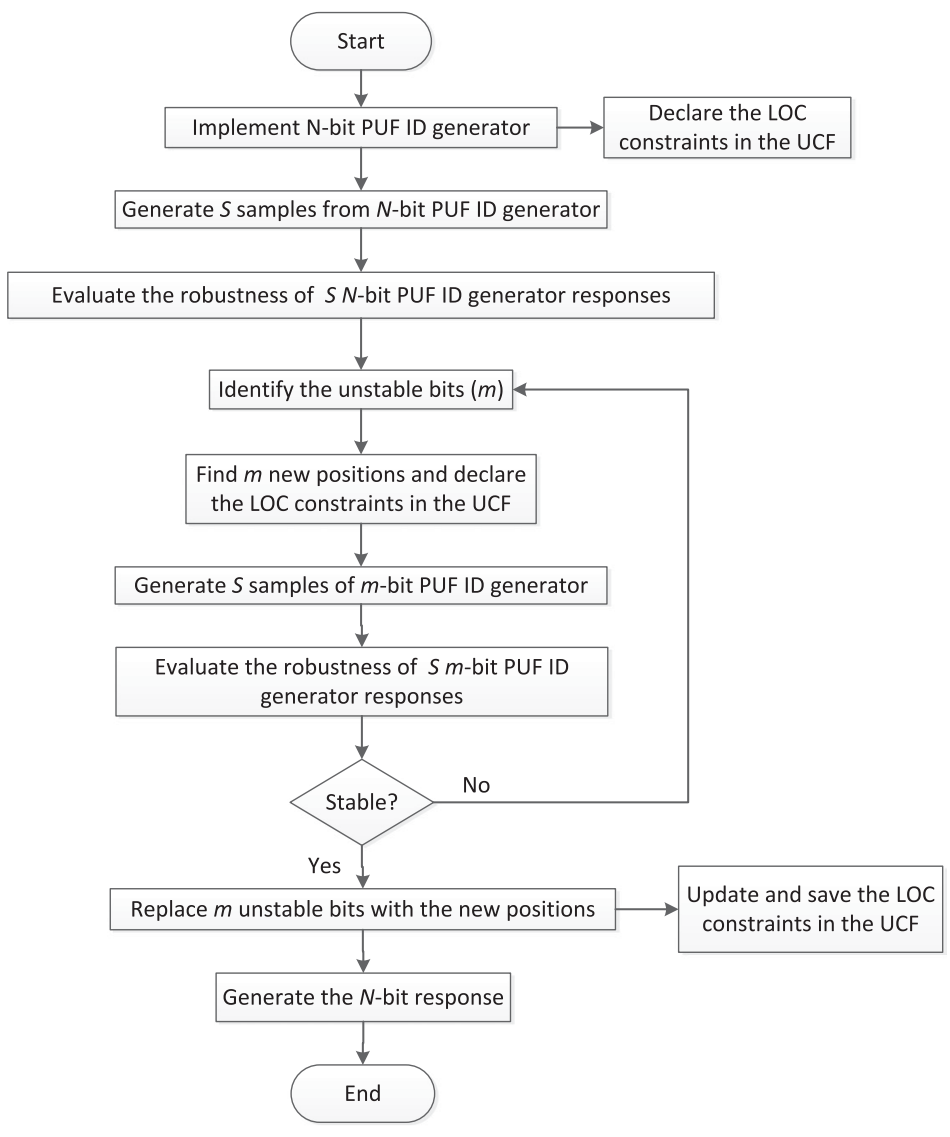

Fig. 7. The flow chart of characterising the floor plan of PUF ID generator IP core architecture.

Xilinx Spartan-6 (CSG324) FPGA device (45nm technology). The 128-bit identification generator design was programmed into 10 identical Spartan-6 LX9 Microboards as shown in Figure 9. One was manually modified to conduct temperature and voltage experiments by varying the core voltage $( \pm 10 \%)$ and the temperature from $25^{\circ} \mathrm{C}$ to $70^{\circ} \mathrm{C}$ as shown in Figure 9.

To evaluate the automated characterisation process, the 128-bit PUF ID design was implemented on a Digilent Nexys4 microboard that comprises a Xilinx Artix-7 XC7A100T FPGA (28nm technology) to prove its feasibility on a more recent technology. The communication and control units on the Xilinx Artix-7 FPGA are similar to those on the Xilinx Spartan-6. One Xilinx Artix-7 FPGA was again modified to conduct temperature and voltage experiments. The core voltage was varied by $\pm 10 \%$ and the temperature from $0^{\circ} \mathrm{C}$ to $75^{\circ} \mathrm{C}$. The core voltage of the Artix-7 FPGA is $1.0 \mathrm{~V}$, which differs from the Spartan-6 FPGA which has a core voltage of $1.2 \mathrm{~V}$.

There are four important metrics used to quantify the performance of a PUF ID generator circuit: uniqueness, reliability, uniformity, and bit-aliasing. These are used to evaluate the improved PUF ID generator design.

\subsection{Uniqueness}

Uniqueness measures inter-chip variation by evaluating how easily a particular PUF ID generator circuit design can differentiate between $k$ different devices. Specifically, it 


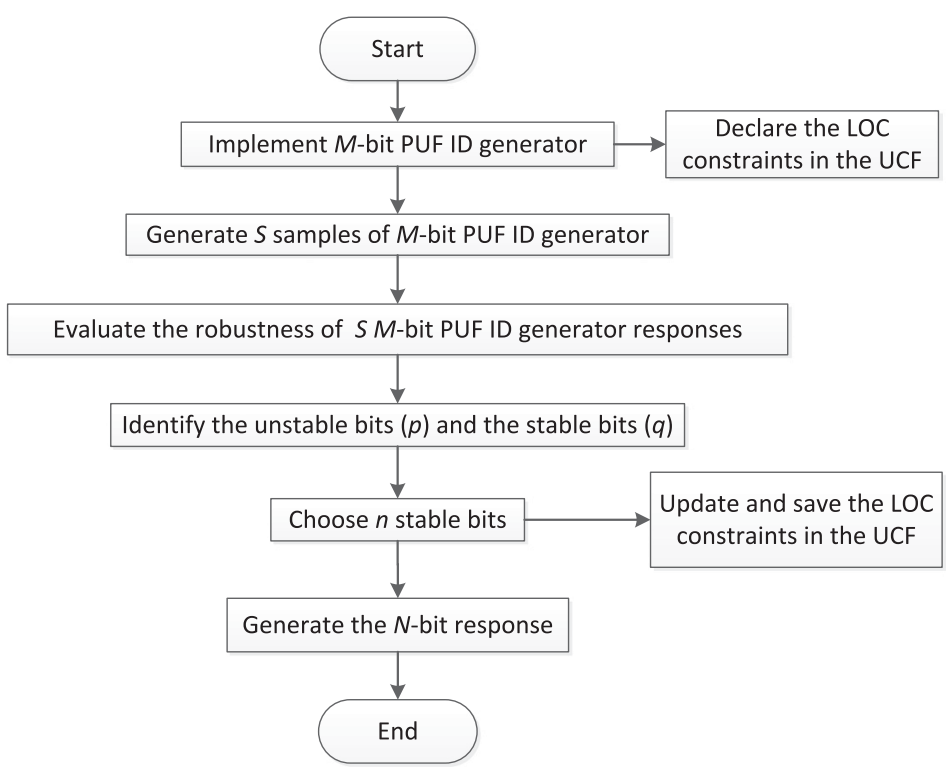

Fig. 8. Flow chart of automated characterisation process.

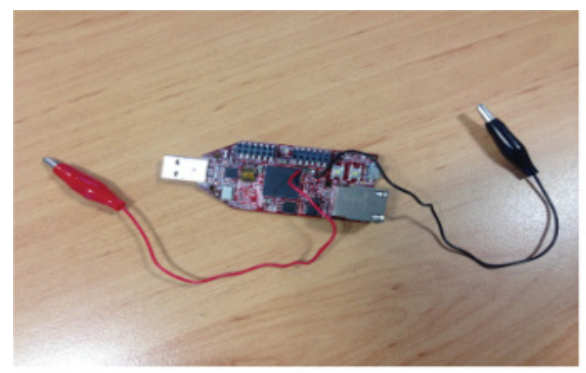

Modified LX9 microboard

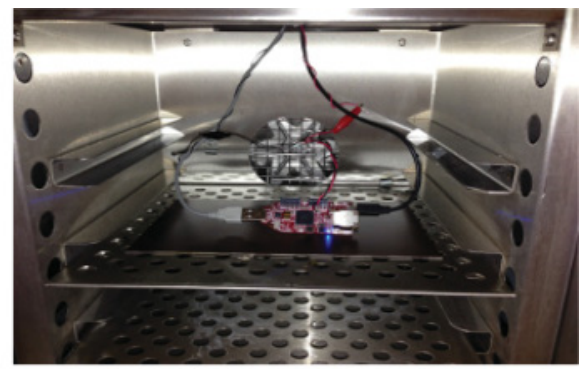

LX9 microboard in heat chamber

Fig. 9. Experimental setup.

quantifies the average inter-chip hamming distances (HDs) between sets of responses extracted from different devices, which implement the same PUF ID generator circuit and have been supplied with the same challenge to show the extent of the difference of responses.

Ideally, when a PUF ID generator circuit is implemented on different devices, it should produce an average inter-chip HD of 50\% when compared between two devices supplied with the same challenge, implying that, on average, half the response bits differ between the two devices even though the same challenge has been used. This is what would be expected should the PUF circuit be replaced with a truly random number.

Accordingly, a percentage figure-of-merit for uniqueness based on average inter-chip HD can be defined. If two chips $i$ and $j$ both implement the same PUF ID generator circuit and have $n$-bit responses $R_{i}$ and $R_{j}$ to the same challenge, $C$, then uniqueness 


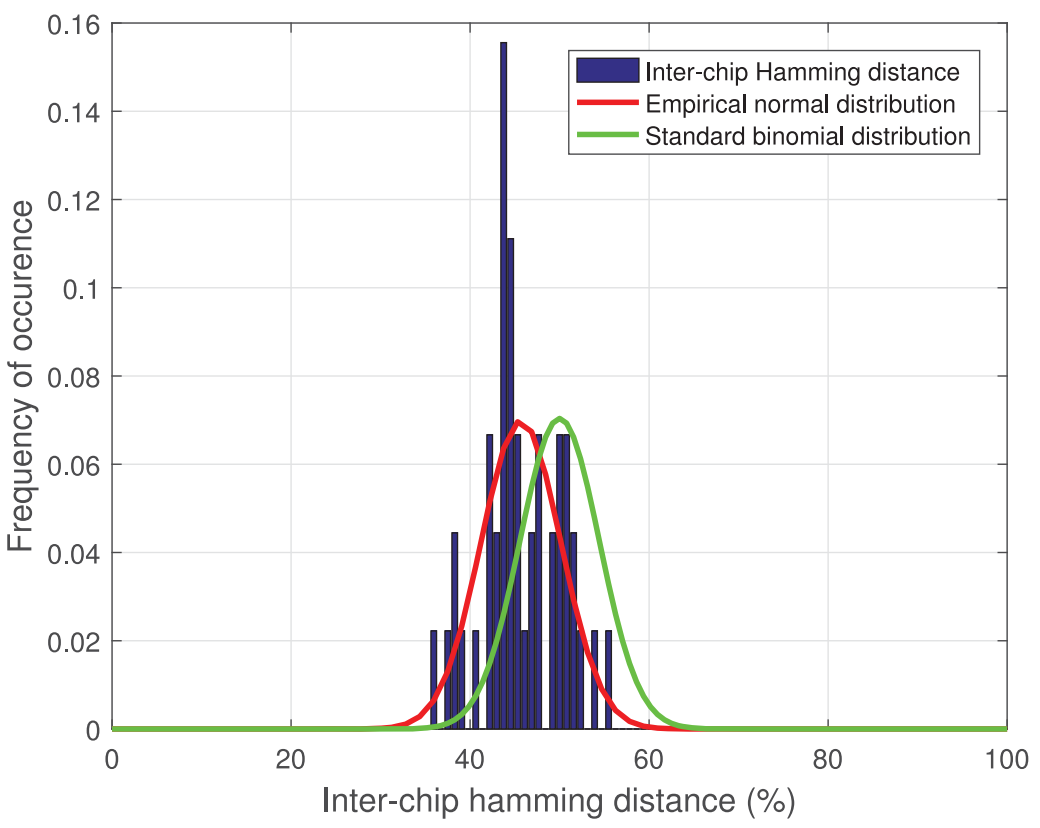

Fig. 10. Uniqueness result.

\section{ALGORITHM 1: Pseudo-Code for Automated Characterisation Process}

\section{1: Phase0: Setup}

2: Declare the position of an $M$-bit PUF ID cell in the UCF, where $M$ is the maximum available slices of the target FPGA

3: $\quad$ Implement $M$-bit PUF ID generator design on the FPGA

4: Phase1: PUF Response

5: for $i=0$ to $S$ do (where $S$ is the sample number for robustness)

6: $\quad$ Generate the response of the $M$-bit PUF ID generator

7: end for

8: Phase2: Evaluate

9: $\quad$ Evaluate the robustness of $S M$-bit PUF ID generator responses

10: Phase3: Identify

11: $\quad$ Identify the unstable bits $(p)$ and the stable bits $(q)$ of the robustness result

12: $\quad$ Note the position of the stable bits $(q)$

\section{3: Phase4: Choose}

14: Randomly choose the position of $n$ stable bits from the $q$ bits as the position of $N$-bit PUF ID

generator, where $N$ is the required bit length of the PUF ID generator

15: Update and declare the position of the $N$-bit PUF ID cell in the UCF

16: Phase5: Generate

17: Generate the response of the $N$-bit PUF ID generator

expressed as the average inter-chip HD among $k$ devices is defined as

$$
\text { Uniqueness }=\frac{2}{k(k-1)} \sum_{i=1}^{k-1} \sum_{j=i+1}^{k} \frac{\operatorname{HD}\left(R_{i}, R_{j}\right)}{N} \times 100 .
$$

A probability density function $(\mathrm{PDF})$ plot of the $\frac{\mathrm{HD}\left(R_{i}, R_{j}\right)}{N} \times 100$ values is shown in Figure 10, where values closer to $50 \%$ indicate better quality uniqueness. This can 


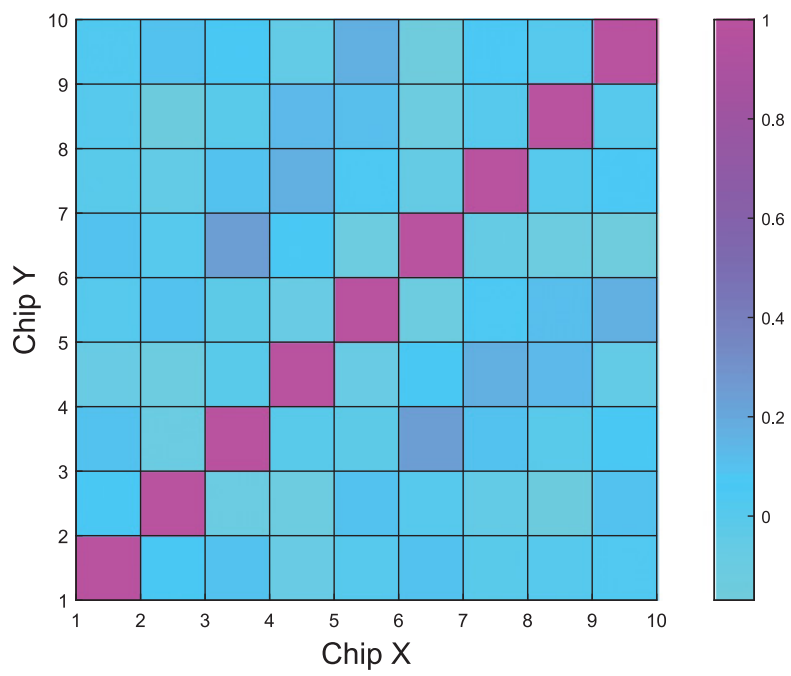

Fig. 11. Cross correlation between different devices.

be approximated as a normal distribution, with an expected uniqueness value of $58.47(45.60 \%)$, with a standard deviation (STD) of $5.71(4.60 \%)$. The red solid line in Figure 10 shows this normal distribution with the empirically determined mean and standard deviation. In the ideal case, viewing the PUF as a random oracle, the uniqueness metric should follow a binomial distribution with a mean of $\frac{n}{2}$ and standard deviation of $\sqrt{\frac{n}{4}}$. This is shown for comparison with the green plot in Figure 10 with a mean of $64(50 \%)$ and standard deviation of $5.6(5 \%)$.

This shows that a uniqueness approaching the ideal value can be expected for the proposed PUF ID generator on this particular set of FPGAs. The uniqueness can also be shown by viewing the cross correlation of the responses from the $n(n=10)$ different devices as shown in Figure 11. The closer the value to 1, the higher the correlation between the responses from device $\mathrm{X}$ and device $\mathrm{Y}$. It can be seen that the crosscorrelation values between the devices are close to 0 , showing that the responses are uncorrelated.

\subsection{Reliability}

Ideally, a given PUF ID generator circuit implemented in any device should be able to perfectly reproduce its output whenever it is queried with a challenge. However, in practice, environmental changes, such as temperature and power supply voltage variations, as well as the natural properties of metastability in PUF ID generator circuits, induce noise in the responses. Therefore, reliability is used to quantify a PUF ID generator's ability to reproduce a response. Reliability can be regarded as a percentage measure of the number of noisy ID response bits.

For a device $i$, reliability is established as a single value by finding the average intrachip HD of $s$ response samples, $R_{i}^{\prime}$, taken at different operating conditions compared to a baseline $N$-bit reference response, $R_{i}$, taken at nominal operating conditions. The average intra-chip HD is estimated as follows:

$$
\mathrm{HD}_{\text {INTRA }}=\frac{1}{s} \sum_{t=1}^{s} \frac{\mathrm{HD}\left(R_{i}, R_{i, t}^{\prime}\right)}{N} \times 100,
$$




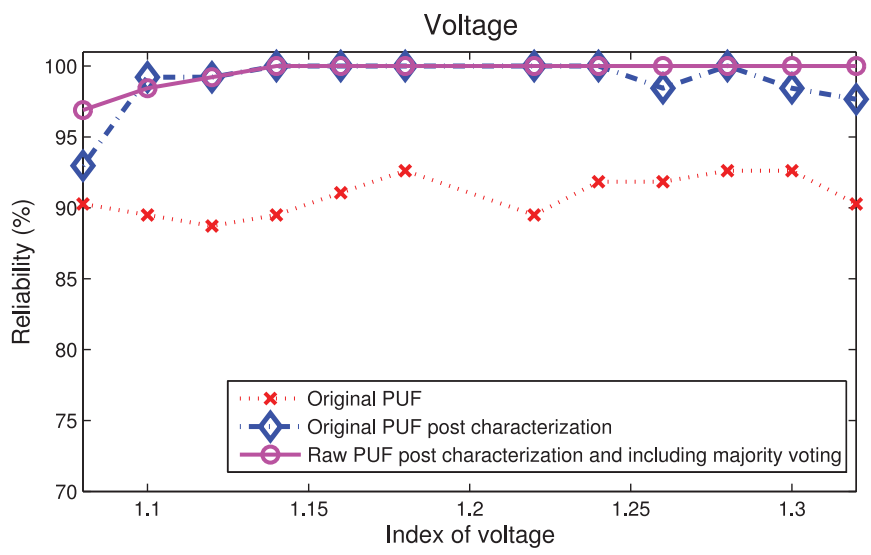

Fig. 12. Reliability comparison over supply voltage variation.

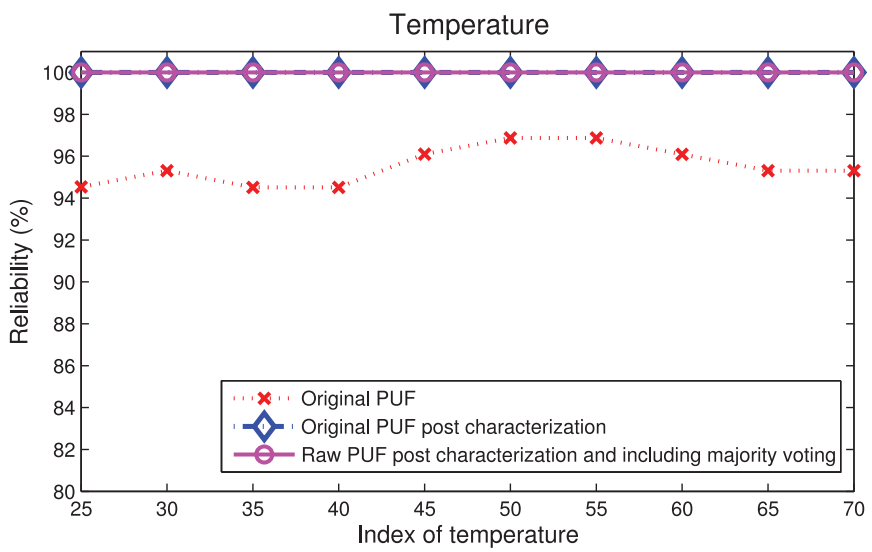

Fig. 13. Reliability comparison over ambient temperature variation.

where $R(i, t)^{\prime}$ is the $t$ th sample of $R_{i}^{\prime}$. The percentage figure of merit for reliability can be defined as

$$
\text { Reliability }=100-\text { HDINTRA. }
$$

Obviously, the ideal value for reliability is $100 \%$. To investigate the reliability of the proposed PUF ID generator design, a 128-bit reference response $R_{i}$ was extracted from a chip $i$ under normal conditions, that is, at room temperature and with normal supply voltage. This is compared with the responses $R_{i}^{\prime}$, taken under varying operating conditions. Note that robustness can be calculated using the same formula as reliability. The only difference is that the response samples for robustness are derived under nominal operating conditions.

6.3.1. Reliability with Manual Characterisation Process. The temperature was varied from $25^{\circ} \mathrm{C}$ to $70^{\circ} \mathrm{C}\left(5^{\circ} \mathrm{C}\right.$ each step) using a convection heat chamber while the core supply voltage was varied by $\pm 10 \% 0.2 \mathrm{~V}$ using a DC-regulated power supply. This covered the permitted operating range of the FPGA and swept all combinations of operating points. Figure 12 and Figure 13 show the results and compare the responses from the previously presented PUF ID generator design, with those from the PUF ID generator design post-characterisation, as well as the PUF ID generator design post-characterisation 


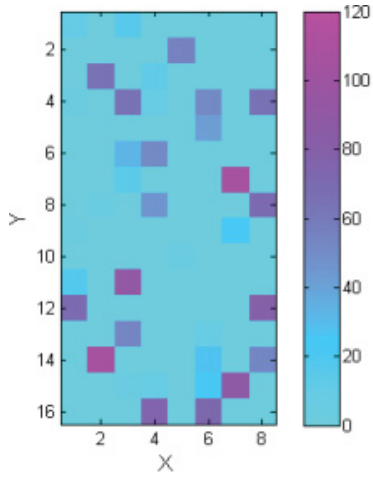

PUF ID generator

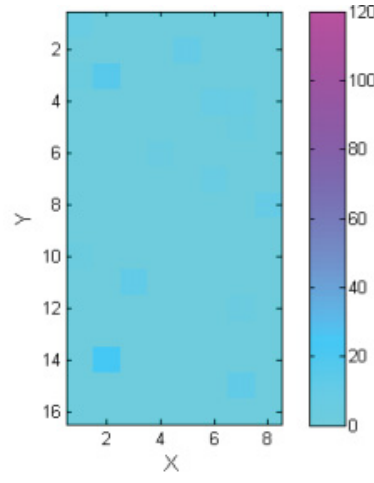

PUF ID generator with post-characterisation

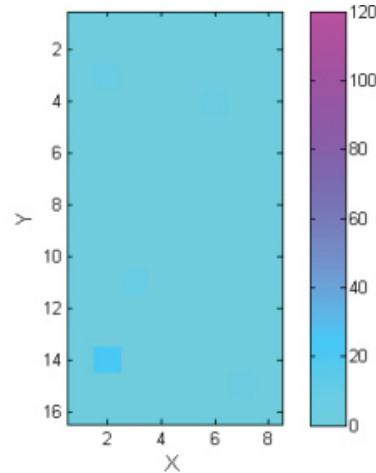

PUF ID generator with post-characterisation and majority function

Fig. 14. Reliability variation maps of 128-bit responses based on the PUF ID generator design, the PUF ID generator design post-characterisation, and the PUF ID generator design post-characterisation with majority function circuitry.

that includes majority function circuit. Over all voltage operating points the improved PUF ID generator designs exhibit a high level of reliability of between $93 \%$ and $100 \%$ for the design post-characterisation, and between $96.5 \%$ and $100 \%$ for the design that employs both characterisation and majority function circuitry. For the temperature results, both improved PUF ID generator designs achieve reliability of $100 \%$ for the FPGAs under evaluation. As expected, the designs that use the characterisation and majority function circuitry are more reliable than the original PUF ID generator design. The average reliability results are listed in Table V. As discussed in the previous section the improvement is due to changing the position of the unreliable 1-bit ID cells which improves the robustness of the response bits.

To estimate the fluctuation in reliability of each bit, the variation of each response bit was compared under normal operating conditions and varying operating conditions. A variation map is used to explain the effects of the ID cell positioning on the response bits. A group of 128-bit responses was extracted under various environmental conditions. The variation from the original response bit is obtained by comparing these responses to the reference response obtained under normal conditions. At each specific position, the difference between the derived response and the reference response can be represented as either " 0 " or " 1 ", where " 0 " means no difference between responses and "1" means the response from the conditioned situation differs from the one obtained under normal conditions. Therefore, the sum of the difference in responses at each position $S_{r_{i, l}}$ can be found, where $r_{i, l}$ denotes the $l$ th position bit on the $i$ th chip. Figure 14 shows the distribution of differences $\left(S_{r_{i, l}}\right)$ from the reliability results for the 128-bit positions. $X$ represents the bit position on the $x$-axis of the floor plan in Figure 3 , and $Y$ represents the bit position on the $y$-axis of the floor plan. The ranges of $X$ and $Y$ are $1 \rightarrow 8$ and $1 \rightarrow 16$, respectively, which indicate the response position as follows:

$$
r_{i, l} \Rightarrow\left\{l_{X}, l_{Y}\right\}
$$

where $l_{X}$ and $l_{Y}$ are the $x$-axis and $y$-axis response position $r_{i, l}$. The subfigures, respectively, show the distribution of the difference on reliability for the PUF ID generator design, the PUF ID generator design using the characterisation process and the PUF ID generator design post-characterisation and including the majority function circuitry. The colorbar ranges are $0 \rightarrow 120$ in Figure 14, where the samples are from 12 differ- 
Table I. BER Results for Voltage Variations for the Original Design, the Design Post-Characterisation, and the Design Post-Characterisation and Including Error Correction Circuitry

\begin{tabular}{|c|c|c|c|c|c|c|c|c|c|c|c|c|c|c|}
\hline & \multicolumn{10}{|c|}{ BER $(\%)$} & SD & Mean \\
$(\%)$
\end{tabular}

Table II. BER Results for Temperature Variations for the Original Design, the Design Post-Characterisation, and the Design Post-Characterisation and Including Error Correction Circuitry

\begin{tabular}{|c|c|c|c|c|c|c|c|c|c|c|c|c|}
\hline \multirow[b]{2}{*}{ Design } & \multicolumn{10}{|c|}{ BER (\%) } & \multirow{2}{*}{$\begin{array}{l}\text { SD } \\
(\%)\end{array}$} & \multirow{2}{*}{$\begin{array}{c}\text { Mean } \\
(\%)\end{array}$} \\
\hline & $25^{\circ} \mathrm{C}$ & $30^{\circ} \mathrm{C}$ & $35^{\circ} \mathrm{C}$ & $40^{\circ} \mathrm{C}$ & $45^{\circ} \mathrm{C}$ & $50^{\circ} \mathrm{C}$ & $55^{\circ} \mathrm{C}$ & $60^{\circ} \mathrm{C}$ & $65^{\circ} \mathrm{C}$ & $70^{\circ} \mathrm{C}$ & & \\
\hline Original & 4.3 & 3.7 & 4.3 & 4.3 & 3.1 & 2.5 & 2.5 & 3.1 & 3.7 & 3.7 & 0.7 & 3.5 \\
\hline CHAR & 0 & 0 & 0 & 0 & 0 & 0 & 0 & 0 & 0 & 0 & 0 & 0 \\
\hline CHAR \& MAJ & 0 & 0 & 0 & 0 & 0 & 0 & 0 & 0 & 0 & 0 & 0 & 0 \\
\hline
\end{tabular}

ent voltages $\times 10$ different temperatures. The darker color indicates that the difference value, $S_{r_{i, l}}$, at position $r_{i, l}$ is larger. In other words, the PUF ID generator exhibits more instability at specific positions. After the manual characterisation process, this instability is reduced. Moreover, using the majority function, the variation of the response is essentially eliminated. It is clear that the characterisation process significantly improves the PUF ID generator design in terms of reliability and by itself provides close to optimal results.

The bit error rate (BER) of the PUF responses for different voltages and temperatures was investigated. Table I and Table II show the BER, standard deviation (SD), and mean (Mean) values across the voltage range from $1.08 \mathrm{~V}$ to $1.32 \mathrm{~V}$ and across the temperature range from $25^{\circ} \mathrm{C}$ to $70^{\circ} \mathrm{C}$. The PUF ID generator design post-characterisation and including temporal majority voting provides the lowest BER, SD, and Mean when the voltage is varied. Interestingly decreasing the core voltage has little effect on the BER. The $S D=0.8 \% \times 128$ bit $=1$ bit, which means that there is variation in just 1 bit of the 128-bit response, and the average BER is $0.4 \%$. The BER, SD, and Mean values of both the design post-characterisation (CHAR) and the design post-characterisation and including error correction circuitry (CHAR \& MAJ) over temperature changes are zero indicating that no errors occur in these designs.

6.3.2. Reliability with Automated Characterisation Process. Initially the robustness of the PUF ID cell design (that is the intra HD of responses from the same device under nominal operating conditions) on an Artix-7 device was calculated for every slice to visualise the variability across a more recent FPGA.

A 128-bit reference response, $R_{i}$, is extracted from a chip $i$ (Artix-7 FPGA) at room temperature and with normal supply voltage. This is compared to the responses $R_{i}^{s}$, taken under the same operating conditions, where $s=1,000$ samples. Equation (2) and Equation (3) are used in calculate robustness. Figure 15 depicts the heat map that shows the stable and unstable bits of a sample chip $i$. The percentage of stable " 0 " bits is $27.12 \%$, the percentage of stable " 1 " bits is $30.75 \%$, and the percentage of the remaining unstable bits is $42.13 \%$. The robustness distribution of stable and unstable bits in the Artix-7 FPGA is shown in Figure 16.

Table III presents detailed robustness results of the stable " 0 " bits, stable " 1 " bits, and the unstable bits on each Artix-7 FPGA and also presents the mean and standard deviation $(S T D)$ values over 10 FPGAs. It can be seen that the distribution of stable "0" bits, stable " 1 " bits, and unstable bits on the 10 FPGAs is very similar and has a very small STD. 


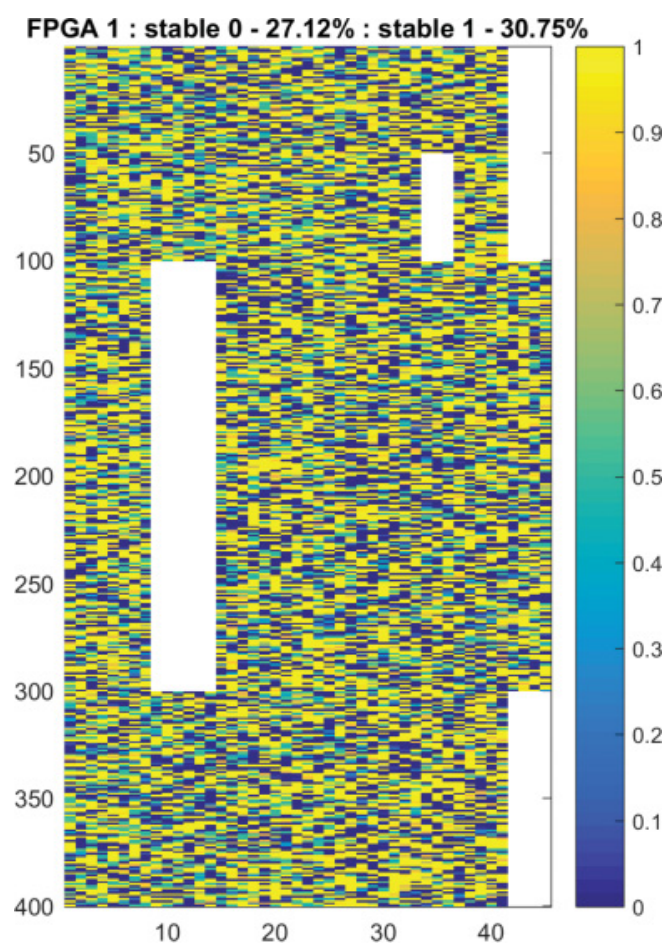

Fig. 15. Heat map of stable and unstable bits of the compact PUF ID generator design on an Artix-7 FPGA.

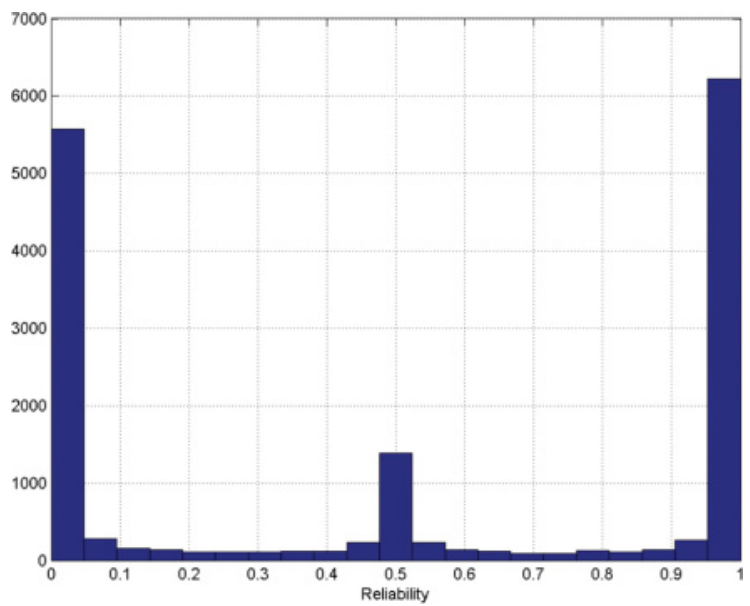

Fig. 16. Robustness distribution of the compact PUF ID generator in Artix-7 FPGA.

To evaluate the reliability of the proposed PUF ID generator design when the automated characterisation process is applied, 128-bit reference responses, $R_{i}$, were extracted from a chip $i$ under normal conditions. These are compared with responses $R_{i}^{\prime}$, taken under varying operating conditions. Temperature was varied from $0^{\circ} \mathrm{C}$ to $75^{\circ} \mathrm{C}$ (in steps of $5^{\circ} \mathrm{C}$ ) using a thermal electric plate while the core supply voltage was varied by $1.0 \mathrm{~V} \pm 10 \%$ using a DC-regulated power supply. This covered the permitted operating range of the FPGA and swept all combinations of operating points. Figure 17 shows the 
Table III. Robustness Results of Stable "0" Bits, Stable "1" Bits, and Unstable Bits on the Ten Artix-7 FPGAs

\begin{tabular}{|c|c|c|c|c|}
\hline FPGA & Bits & Stable 0's & Stable 1's & Unstable \\
\hline 1 & 15,850 & $4,299(27.12 \%)$ & $4,874(30.75 \%)$ & $6,677(42.12 \%)$ \\
\hline 2 & 15,850 & $4,951(31.23 \%)$ & $4,388(27.68 \%)$ & $6,511(41.07 \%)$ \\
\hline 3 & 15,850 & $4,579(28.89 \%)$ & $4,728(29.83 \%)$ & $6,543(41.28 \%)$ \\
\hline 4 & 15,850 & $4,242(26.76 \%)$ & $4,831(30.47 \%)$ & $6,777(42.75 \%)$ \\
\hline 5 & 15,850 & $4,986(31.45 \%)$ & $4,832(30.48 \%)$ & $6,032(38.05 \%)$ \\
\hline 6 & 15,850 & $5,505(34.73 \%)$ & $5,492(34.65 \%)$ & $4,853(30.61 \%)$ \\
\hline 7 & 15,850 & $4,338(27.36 \%)$ & $4,582(28.90 \%)$ & $6,930(43.72 \%)$ \\
\hline 8 & 15,850 & $4,048(25.53 \%)$ & $5,080(32.05 \%)$ & $6,722(42.41 \%)$ \\
\hline 9 & 15,850 & $4,262(26.89 \%)$ & $5,113(32.25 \%)$ & $6,475(40.85 \%)$ \\
\hline 10 & 15,850 & $5,572(35.15 \%)$ & $4,041(25.49 \%)$ & $6,237(39.35 \%)$ \\
\hline Mean & & $4,678(29.51 \%)$ & $4,796(30.25 \%)$ & $6,376(40.22 \%)$ \\
\hline STD & & $546(3.44 \%)$ & $403(2.54 \%)$ & $595(3.75 \%)$ \\
\hline All & & $1(0.006 \%)$ & $0(0.000 \%)$ & $15,849(99.99 \%)$ \\
\hline
\end{tabular}
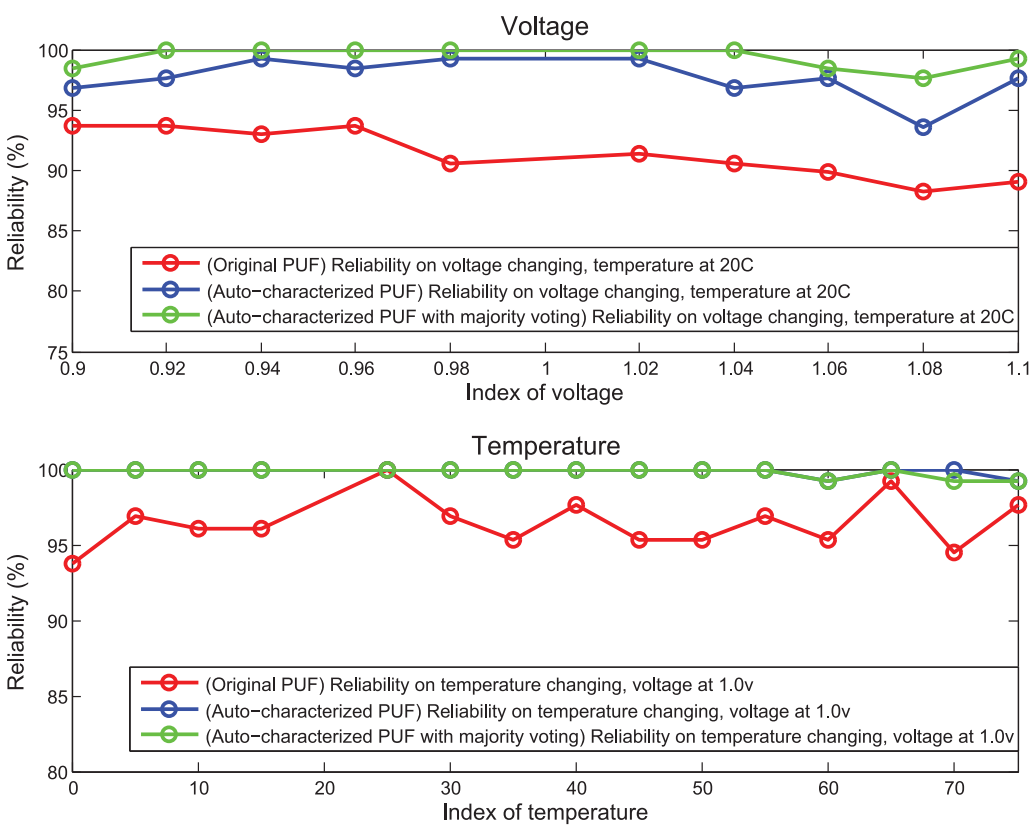

Fig. 17. Reliability results of 128-bit PUF ID generator based on the original PUF ID generator design, the PUF ID generator design post auto-characterisation, and the PUF ID generator design post autocharacterisation with temporal majority voting.

results and compares the responses from the PUF ID generator design, with those from the PUF ID generator design post auto-characterisation as well as the PUF ID generator design post auto-characterisation that includes temporal majority voting. Over all voltage operating points, the improved PUF ID generator designs exhibit a high level of reliability of between $95 \%$ and $100 \%$ for the design post auto-characterisation and between $97 \%$ and $100 \%$ for the design that employs both auto-characterisation and temporal majority voting. For the temperature results, both improved PUF ID generator designs achieve reliability close to $100 \%$. 
Table IV. Reliability Results of Manual and Automated Characterisation Processes on Spartan-6 and Artix-7 FPGAs

\begin{tabular}{|c|c|c|c|c|c|c|c|c|c|}
\hline FPGA & \multicolumn{3}{|c|}{ Over voltage variation $(\%)$} & \multicolumn{3}{|c|}{ Over temperature variation (\%) } & \multicolumn{3}{|c|}{ Average $(\%)$} \\
\hline \multirow[t]{2}{*}{ Spartan6 } & Original & $\begin{array}{c}\text { Manual } \\
\text { CHAR }\end{array}$ & $\begin{array}{c}\text { Manual } \\
\text { CHAR+MAJ }\end{array}$ & Original & $\begin{array}{c}\text { Manual } \\
\text { CHAR }\end{array}$ & $\begin{array}{c}\text { Manual } \\
\text { CHAR+MAJ }\end{array}$ & Original & $\begin{array}{r}\text { Manual } \\
\text { CHAR }\end{array}$ & $\begin{array}{c}\text { Manual } \\
\text { CHAR+MAJ }\end{array}$ \\
\hline & $90.87 \%$ & $98.83 \%$ & $99.55 \%$ & $95.54 \%$ & $100 \%$ & $100 \%$ & $93.21 \%$ & $99.42 \%$ & $99.78 \%$ \\
\hline \multirow[t]{2}{*}{ Artix7 } & Original & $\begin{array}{c}\text { Auto } \\
\text { CHAR }\end{array}$ & $\begin{array}{c}\text { Manual } \\
\text { CHAR+MAJ }\end{array}$ & Original & $\begin{array}{l}\text { Auto } \\
\text { CHAR }\end{array}$ & $\begin{array}{c}\text { Auto } \\
\text { CHAR+MAJ }\end{array}$ & Original & $\begin{array}{c}\text { Auto } \\
\text { CHAR }\end{array}$ & $\begin{array}{c}\text { Auto } \\
\text { CHAR+MAJ }\end{array}$ \\
\hline & $91.40 \%$ & $97.58 \%$ & $99.35 \%$ & $96.46 \%$ & $99.89 \%$ & $99.84 \%$ & $93.93 \%$ & $98.74 \%$ & $99.60 \%$ \\
\hline
\end{tabular}

Table V. PUF ID Generator Results of the Original Design, The Design Post-Characterisation, and the Design PostCharacterisation and Including Error Correction Circuitry

\begin{tabular}{|c|c|c|c|}
\hline Metrics & Original & CHAR & CHAR \& MAJ \\
\hline Uniqueness & $48.52 \%$ & $45.60 \%$ & $45.60 \%$ \\
\hline Reliability & $92.00 \%$ & $98.97 \%$ & $99.58 \%$ \\
\hline Uniformity & $51.06 \%$ & $50.60 \%$ & $50.54 \%$ \\
\hline Non-bit-aliasing & $56.48 \%$ & $56.48 \%$ & $56.48 \%$ \\
\hline
\end{tabular}

A comparison of the improved designs post-manual characterisation and postautomated characterisation are provided in Table IV. It is clear from the results that the post-characterisation processes are an effective way to improve the reliability of the PUF ID generator design.

\subsection{Uniformity}

The uniformity of a PUF ID generator circuit measures the proportion of binary ones and zeros in a response, that is, a one-to-zero ratio, and the likelihood of each value. If a response possesses ideal uniformity and is truly random, then the distribution of bit values will be $50 \%$ ones and zeros. Having this property is required from a security perspective to prevent an attacker from guessing if a response of a particular device is biased towards a particular value. To estimate uniformity is simply a matter of finding the hamming weight (HW) of a response, which will reveal the proportion of ones and zeros, and any biases.

For device $i$ and an $n$-bit response, the percentage HW of the $n$-bit response is given as follows:

$$
(\mathrm{HW})_{l}=\frac{1}{n} \sum_{l=1}^{n} r_{i, l} \times 100,
$$

where $r_{i, l}$ is the $l$ th position of the response bit on the $i$ th chip.

The proportion of 0's and 1's in a response is expected to be close to 50\%, and in this work the response uniformity is $51.06 \%$ for the original PUF ID generator design, $50.60 \%$ for the PUF ID generator design post-characterisation, and 50.54\% for the PUF ID generator design post-characterisation and including the majority function circuitry, as shown in Table V.

\subsection{Bit Aliasing}

An effective PUF ID generator design should not exhibit bit-aliasing when implemented on different devices. Bit-aliasing is when the ID response at stable positions on different chips is identical or almost identical. To determine if bit-aliasing occurs, the total number of $0 \mathrm{~s}$ and $1 \mathrm{~s}$ in a response from the same $p$ th position of $k$ devices is calculated 
using the HW as follows:

$$
(\mathrm{HW})_{p}=\frac{1}{k} \sum_{i=1}^{k} r_{i, p} \times 100,
$$

where $r_{p, i}$ is the $p$ th position of a response bit on the $i$ th chip.

If bit-aliasing occurs and different devices generate the same response from many physical positions, then the security guarantees no longer hold. The percentage of $0 \mathrm{~s}$ and $1 \mathrm{~s}$ at the same position in 10 chips $(k=10)$ is evaluated and is shown in Table V. The value for each of the designs is $56.48 \%$, which means that all of the bit positions are sufficiently different such that bit-aliasing is avoided for all three PUF ID generator designs.

Table V lists the results of all the evaluated PUF ID generator metrics. As previously mentioned, the reliability result has significantly improved using characterisation and a majority function and is very close to the ideal value of $100 \%$, with the uniformity also improving. The uniqueness decreases slightly using the improved PUF ID generator design; however, this could be optimized by changing the position of the 1-bit ID cell hard-macro to also balance the percentage of $1 \mathrm{~s}$ and $0 \mathrm{~s}$ in each response. Bit-aliasing does not occur in any of the designs.

\subsection{Hardware Resources and Performance Analysis}

For the manual characterisation process, 10 identical Xilinx Spartan-6 boards were tested, each assembled with identical parts and components. There are a total of 1,430 slices on a Spartan-6 LX9 FPGA, where each slice contains four LUTs and eight flip-flops. Each ID bit response generation design only needs one slice; hence, our 128-bit identification generator without error correction circuitry occupies only $\frac{128}{1430} \times 100 \%=8.95 \%$ of the total slice resource, and $\frac{128+128}{1430}=17.90 \%$ with error correction circuitry. As can be seen the resource usage is minimal, even on a small FPGA. The layout is controlled using hard macros, which helps achieve the minimal resource footprint.

For the automated characterisation process, 10 identical Xilinx Artix-7 boards were tested. Although a Xilinx Artix-7 FPGA does not have a SLICEX, its SLICEL and SLICEM are essentially the same as that of a Xilinx Spartan-6. The SLICEL and SLICEM of the Xilinx Artix-7 FPGA include all of the components of a SLICEX; hence the 1-bit PUF ID generator can be implemented on either the SLICEM or SLICEL also. In this experiment, a SLICEL is used to implement the hard macro. There are a total of 15, 850 slices on a Xilinx Artix-7 XC7A100T FPGA. Each 1-bit PUF ID generator design only needs one slice; hence, the 128-bit PUF ID generator design without temporal majority voting circuitry occupies only $\frac{128}{15,850} \times 100 \%=0.81 \%$ of the total slice resource available on this FPGA.

The resource usage comparison between the proposed PUF ID generator and other Weak PUFs implemented on hardware devices is shown in Table VI. The SRAM PUF proposed by Guajardo et al. [2007], using a SRAM memory cell can return a response on power-up. The Latch PUF proposed by Su et al. [2008] is implemented on an ASIC not FPGA. The flip-flop PUF [Maes et al. 2008], similar to SRAM PUF, uses the powerup values of the flip-flops; however, its randomness is limited, and post-processing is required. The Butterfly PUF [Kumar et al. 2008], which is also suitable for FPGA implementation, as it can be implemented using basic logic gates, reported $94 \%$ reliability over temperature variations. However, reliability over voltage changes is not provided. It consumes 130 slices of a Xilinx Virtex-5 FPGA device for a 64-bit response generation and hence uses twice the hardware resources of the proposed 1-bit PUF ID generator 
Table VI. Comparison of Hardware Resource Consumption and Metrics of Different Weak PUF Designs

\begin{tabular}{|c|c|c|c|c|c|}
\hline PUF design & $\mathrm{U}^{\prime}$ & $\mathrm{R}^{\prime}$ & Hardware & Resp (bit) & Consumption \\
\hline $\begin{array}{l}\text { SRAM PUF [Guajardo et al. } \\
\text { 2007] }\end{array}$ & $49.97 \%$ & $>88 \%{ }^{t}$ & FPGA & 128 & $\begin{array}{l}4,600 \text { SRAM } \\
\text { memory bits }\end{array}$ \\
\hline Latch PUF [Su et al. 2008] & $50.55 \%$ & $96.96 \%$ & $\begin{array}{l}0.13 \text { um } \\
\text { CMOS }\end{array}$ & 128 & $\begin{array}{l}1 \text { latch for } \\
\text { each ID cell }\end{array}$ \\
\hline $\begin{array}{l}\text { Latch PUF [Yamamoto et al. } \\
\text { 2011] }\end{array}$ & $46 \%$ & $>87 \%{ }^{t}$ & Spartan 3 & 128 & $2 \times 128$ slices \\
\hline Flip-flop PUF [Maes et al. 2008] & $\approx 50 \% *$ & $>95 \% v$ & Virtex 2 & 4,096 & 4,096 flip-flops \\
\hline $\begin{array}{l}\text { Flip-flop PUF [van der Leest } \\
\text { et al. 2010] }\end{array}$ & $36 \%$ & $>87 \%^{t}$ & ASIC & 1,024 & 1,024 flip-flops \\
\hline $\begin{array}{l}\text { Buskeeper PUF [Simons et al. } \\
\text { 2012] }\end{array}$ & $49 \%$ & $\begin{array}{l}>80 \%{ }^{t}, \\
>95 \% v\end{array}$ & $\begin{array}{c}\text { TSMC } 65 \\
\mathrm{~nm}\end{array}$ & 192 & $1 G E^{1}$ \\
\hline $\begin{array}{l}\text { Butterfly PUF [Kumar et al. } \\
2008]\end{array}$ & $\approx 50 \% *$ & $94 \%$ & Virtex 5 & 64 & 130 slices \\
\hline RO PUF [Suh and Devadas 2007] & $46.15 \%$ & $99.52 \%$ & Virtex 4 & 128 & $16 \times 64$ array $^{2}$ \\
\hline CRO PUF [Merli et al. 2010] & $43.50 \%$ & $\begin{array}{l}>96 \%{ }^{t}, \\
\approx 100 \%^{*}\end{array}$ & Spartan 3 & 127 & $\begin{array}{c}64 \text { slices for } \\
\text { ROs except } \\
\text { counters }\end{array}$ \\
\hline $\begin{array}{l}\text { PUF ID generator [Gu et al. } \\
2014]\end{array}$ & $\begin{array}{l}48.52 \%(\mathrm{~S}-6), \\
49.90 \%(\mathrm{~A}-7)\end{array}$ & $\begin{array}{l}93.21 \%(\mathrm{~S}-6), \\
93.93 \%(\mathrm{~A}-7)\end{array}$ & $\begin{array}{l}\text { Spartan 6, } \\
\text { Artix } 7\end{array}$ & 128 & 128 slices \\
\hline $\begin{array}{l}\text { Ultra-compact PUF ID generator } \\
\text { [Gu and O'Neill 2015] }\end{array}$ & $49.93 \%$ & $93.96 \%$ & Spartan 6 & 128 & 40 slices \\
\hline $\begin{array}{c}\text { Proposed improved PUF ID } \\
\text { generator }\end{array}$ & $45.60 \%(\mathrm{~S}-6)$ & $\begin{array}{l}99.42 \%(\mathrm{M}), \\
98.74 \%(\mathrm{~A})\end{array}$ & $\begin{array}{l}\text { Spartan } 6, \\
\text { Artix } 7\end{array}$ & 128 & 128 slices \\
\hline
\end{tabular}

${ }^{1} \mathrm{GE}$ represented gate equivalent. ${ }^{2} 16 \times 64$ array $=1024 \mathrm{ROs}$; each RO consisting of five inverters and one AND. U' is uniqueness, R' is reliability. Resp is response. ${ }^{t}$ under temperature variation. ${ }^{v}$ under supply voltage variation. * required post-processing. $\mathrm{M}$ is manual characterisation process, $\mathrm{A}$ is automated characterisation process.

design. The RO PUFs [Merli et al. 2010; Suh and Devadas 2007] and the CRO PUF [Merli et al. 2010] have been implemented on different FPGAs, for example, Xilinx Virtex-4 and Spartan-3. The hardware resource consumption is at least 384 slices for a 64-bit response. It can be seen that the proposed PUF ID generator design is the most lightweight FPGA-based Weak PUF design reported to date. Moreover, the performance results for uniqueness and reliability show the effectiveness of the proposed PUF design.

\section{CONCLUSIONS}

In this article, we have shown that an effective, reliable, and low-cost PUF ID generator design is achievable for an FPGA device. A single ID cell fits efficiently within one FPGA slice and can be tailored for instantiation as a hard-macro to achieve balanced routing. The design is the most compact FPGA-based Weak PUF architecture reported to date. An example 128-bit PUF ID generator is implemented on both a Xilinx Spartan-6 and Artix-7 FPGA. It utilizes only half a slice for each 1-bit ID cell that consumes only $8.95 \%$ of the overall hardware resources of the Spartan- 6 device and $0.81 \%$ of the Artix7 device. The manual characterisation post-processing enhances the reliability of the 128-bit PUF ID generator design from $93.21 \%$ to $99.42 \%$ without the requirement of any additional hardware resources. A further improvement to $99.78 \%$ is achieved when temporal majority voting is also employed. An automated characterisation method is presented, which improves the reliability of the 128-bit PUF ID generator from $93.93 \%$ to $98.74 \%$ without the requirement of any additional hardware resources, and $99.60 \%$ for the design that employs a temporal majority voting. Overall, experimental results demonstrate high uniqueness, reliability, uniformity, and no bit-aliasing with values of $45.60 \%, 50.60 \%$, and $56.48 \%$ using characterisation process and values of $45.60 \%$, $50.54 \%$, and $56.58 \%$ using a further temporal majority voting. 


\section{REFERENCES}

Georg T. Becker. 2015. The Gap Between Promise and Reality: On the Insecurity of XOR Arbiter PUFs. Springer, Berlin, 535-555. DOI : http://dx.doi.org/10.1007/978-3-662-48324-4_27

Mudit Bhargava and Ken Mai. 2014. An efficient reliable PUF-based cryptographic key generator in 65nm CMOS. In Proceedings of the ACM /IEEE Design, Automation and Test in Europe Conference and Exhibition (DATE'14). 1-6. DOI : http://dx.doi.org/10.7873/DATE.2014.083

Christoph Bohm, Maximilian Hofer, and Wolfgang Pribyl. 2011. A microcontroller SRAM-PUF. In Proceedings of the 5th International Conference on Network and System Security (NSS'11). 269-273.

Leonid Bolotnyy and Gabriel Robins. 2007. Physically unclonable function-based security and privacy in RFID systems. In Proceedings of the 5th Annual IEEE International Conference on Pervasive Computing and Communications, 2007 (PerCom'07). IEEE, 211-220.

Qingqing Chen, Gyorgy Csaba, Paolo Lugli, Ulf Schlichtmann, and Ulrich Ruhrmair. 2011. The bistable ring PUF: A new architecture for strong physical unclonable functions. In Proceedings of the IEEE International Symposium on Hardware-Oriented Security and Trust (HOST'11). 134-141. DOI : http://dx.doi.org/10.1109/HST.2011.5955011

Mafalda Cortez, Said Hamdioui, Vincent van der Leest, Roel Maes, and Geert-Jan Schrijen. 2013. Adapting voltage ramp-up time for temperature noise reduction on memory-based PUFs. In Proceedings of the IEEE International Symposium on Hardware-Oriented Security and Trust (HOST'13). 35-40. DOI : http://dx.doi.org/10.1109/HST.2013.6581562

Jeroen Delvaux and Ingrid Verbauwhede. 2014. Fault injection modeling attacks on $65 \mathrm{~nm}$ arbiter and RO sum PUFs via environmental changes. IEEE Trans. Circuits Syst. I, Reg. Papers 61, 6 (June 2014), 1701-1713. DOI : http://dx.doi.org/10.1109/TCSI.2013.2290845

Achiranshu Gary and Tony T. Kim. 2014. Design of SRAM PUF with improved uniformity and reliability utilizing device aging effect. In Proceedings of the IEEE International Symposium on Circuits and Systems (ISCAS'14). IEEE, 1941-1944.

Blaise Gassend, Dwaine Clarke, Marten van Dijk, and Srinivas Devadas. 2002. Silicon physical random functions. In Proceedings of the 9th ACM Conference on Computer and Communications Security (CCS'02). ACM, New York, NY, 148-160. DOI : http://dx.doi.org/10.1145/586110.586132

Chongyan Gu, Yijun Cui, Neil Hanley, and Máire O’Neill. 2016. Novel lightweight FF-APUF design for FPGA. In Proceedings of 29th IEEE International System-on-Chip Conference, (SOCC'16).

Chongyan Gu, Julian Murphy, and Máire O’Neill. 2014. A unique and robust single slice FPGA identification generator. In Proceedings of the IEEE International Symposium on Circuits and Systems (ISCAS'14). 1223-1226. DOI : http://dx.doi.org/10.1109/ISCAS.2014.6865362

Chongyan Gu and Máire O’Neill. 2015. Ultra-compact and robust FPGA-based PUF identification generator. In Proceedings of the IEEE International Symposium on Circuits and Systems (ISCAS'15). DOI : http://dx.doi.org/10.1109/ISCAS.2015.7168788

Jorge Guajardo, Sandeep S. Kumar, Geert-Jan Schrijen, and Pim Tuyls. 2007. FPGA Intrinsic PUFs and Their Use for IP Protection. Springer, Berlin, 63-80 pages. DOI : http://dx.doi.org/10.1007/978-3-540-74735-2_5

Charles Herder, Meng-Day Yu, Farinaz Koushanfar, and Srinivas Devadas. 2014. Physical unclonable functions and applications: A tutorial. Proc. IEEE 102, 8 (2014), 1126-1141.

Daniel E. Holcomb, Wayne P. Burleson, and Kevin Fu. 2009. Power-up SRAM state as an identifying fingerprint and source of true random numbers. IEEE Trans. Comput 58, 9 (2009), 1198-1210.

Daniel E. Holcomb, Amir Rahmati, Mastooreh Salajegheh, Wayne P. Burleson, and Kevin Fu. 2013. DRVFingerprinting: Using Data Retention Voltage of SRAM Cells for Chip Identification. Springer, Berlin, 165-179. DOI : http://dx.doi.org/10.1007/978-3-642-36140-1_12

Yohei Hori, Hyunho Kang, Toshihiro Katashita, Akashi Satoh, Shinichi Kawamura, and Kazukuni Kobara. 2014. Evaluation of physical unclonable functions for $28-\mathrm{nm}$ process field-programmable gate arrays. $J$. Inf. Process. 22, 2 (2014), 344-356.

Intrisic-ID. accessed 17th June 2015. NXP Secures Over Two Billion Payment and Government ID Cards with SmartMX. Retrieved June 17, 2015 from https://www.intrinsic-id.com/nxp-secures-over-twobillion-payment-and-government-id-cards-/with-smartmx/.

Raghavan Kumar and Wayne Burleson. 2014. On design of a highly secure PUF based on non-linear current mirrors. In Proceedings of the IEEE International Symposium on Hardware-Oriented Security and Trust (HOST'14). IEEE Computer Society, 38-43. DOI : http://dx.doi.org/10.1109/HST.2014.6855565

Sandeep S. Kumar, Jorge Guajardo, Roel Maes, G.-J. Schrijen, and Pim Tuyls. 2008. The butterfly PUF protecting IP on every FPGA. In Proceedings of the IEEE International Symposium on HardwareOriented Security and Trust (HOST'08). 67-70. DOI : http://dx.doi.org/10.1109/HST.2008.4559053 
Klaus Kursawe, Ahmad-Reza Sadeghi, Dries Schellekens, Boris Skoric, and Pim Tuyls. 2009. Reconfigurable physical unclonable functions - enabling technology for tamper-resistant storage. In Proceedings of the IEEE International Workshop on Hardware-Oriented Security and Trust (HST'09). IEEE Computer Society, Washington, DC, 22-29. DOI : http://dx.doi.org/10.1109/HST.2009.5225058

Daihyun Lim, Jae W. Lee, Blaise Gassend, G. Edward Suh, Marten Van Dijk, and Srinivas Devadas. 2005. Extracting secret keys from integrated circuits. IEEE Trans. VLSI Syst 13, 10 (2005), 1200-1205.

Roel Maes, Pim Tuyls, and Ingrid Verbauwhede. 2008. Intrinsic PUFs from flip-flops on reconfigurable devices. In Proceedings of the 3rd Benelux Workshop on Information and System Security (WISSec'08). Eindhoven, NL, 17.

Ahmed Mahmoud, Ulrich Rührmair, Mehrdad Majzoobi, and Farinaz Koushanfar. 2013. Combined modeling and side channel attacks on strong PUFs. IACR Cryptology ePrint Archive 2013 (2013), 632. http://eprint.iacr.org/2013/632.

Abhranil Maiti, Inyoung Kim, and Patrick Schaumont. 2012. A robust physical unclonable function with enhanced challenge-response set. IEEE Trans. Inf. Forens. Secur. 7 (2012), 333-345.

Abhranil Maiti and Patrick Schaumont. 2012. A novel microprocessor-intrinsic physical unclonable function. In Proceedings of the 22nd International Conference on Field Programmable Logic and Applications (FPL'12). 380-387. DOI : http://dx.doi.org/10.1109/FPL.2012.6339208

Mehrdad Majzoobi, Akshat Kharaya, Farinaz Koushanfar, and Srinivas Devadas. 2014. Automated design, implementation, and evaluation of arbiter-based PUF on FPGA using programmable delay lines. IACR Cryptology ePrint Archive 2014 (2014), 639.

Dominik Merli, Frederic Stumpf, and Claudia Eckert. 2010. Improving the quality of ring oscillator PUFs on FPGAs. In Proceedings of the 5th Workshop on Embedded Systems Security (WESS'10). ACM, New York, NY, Article 9, 9 pages. DOI : http://dx.doi.org/10.1145/1873548.1873557

Microsemi. Microsemi SmartFusion2 SoC FPGAs Offer More Resources in Low Density Devices With The Lowest Power, Proven Security and Exceptional Reliability. Retrieved June 17, 2015 from http://www.microsemi.com/products/fpga-soc/soc-fpga/smartfusion2.

Julian Murphy, Máire O’Neill, Frank Burns, Alex Bystrov, Alexandre Yakovlev, and Basel Halak. 2012. Selftimed physically unclonable functions. In Proceedings of the 5th IFIP International Conference on New Technologies, Mobility and Security (NTMS'12). 1-5.

Ravikanth Pappu, Ben Recht, Jason Taylor, and Neil Gershenfeld. 2002. Physical one-way functions. Science 297, 5589 (2002), 2026-2030.

Ed Peterson. 2015. Leveraging Asymmetric Authentication to Enhance Security-Critical Applications Using Zynq-7000 All Programmable SoCs. Retrieved October 2015 from http:/www.xilinx.com/support/ documentation/white_papers/wp468_asym-auth-zynq-7000.pdf.

Ulrich Rührmair, Frank Sehnke, Jan Sölter, Gideon Dror, Srinivas Devadas, and Jürgen Schmidhuber. 2010. Modeling attacks on physical unclonable functions. In Proceedings of the 17th ACM Conference on Computer and Communications Security (CCS'10). ACM, New York, NY, 237-249. DOI : http://dx.doi.org/10.1145/1866307.1866335

Peter Simons, Erik van der Sluis, and Vincent van der Leest. 2012. Buskeeper PUFs, a promising alternative to D flip-flop PUFs. In Proceedings of the IEEE International Symposium on Hardware-Oriented Security and Trust (HOST'12). 7-12. DOI : http://dx.doi.org/10.1109/HST.2012.6224311

Nicolas Sklavos. 2013. Securing Communication Devices via Physical Unclonable Functions (PUFs). Springer, Wiesbaden, 253-261. DOI : http://dx.doi.org/10.1007/978-3-658-03371-2_22

Ying Su, Jeremy Holleman, and Brian P. Otis. 2008. A digital 1.6 pJ/bit chip identification circuit using process variations. IEEE J. Solid-State Circ. 43 (2008), 69-77.

G. Edward Suh and Srinivas Devadas. 2007. Physical unclonable functions for device authentication and secret key generation. In Proceedings of the 44th Annual Design Automation Conference (DAC'07). ACM, New York, NY, 9-14. DOI : http://dx.doi.org/10.1145/1278480.1278484

Vincent van der Leest, Geert-Jan Schrijen, Helena Handschuh, and Pim Tuyls. 2010. Hardware intrinsic security from D flip-flops. In Proceedings of the 5th ACM Workshop on Scalable Trusted Computing (STC'10). ACM, New York, NY, 53-62. DOI : http://dx.doi.org/10.1145/1867635.1867644

Arunkumar Vijayakumar and Sandip Kundu. 2015. A novel modeling attack resistant PUF design based on non-linear voltage transfer characteristics. In Proceedings of the 2015 Design, Automation \& Test in Europe Conference \& Exhibition (DATE'15), Wolfgang Nebel and David Atienza (Eds.). ACM, 653-658. http://dl.acm.org/citation.cfm?id=2755903

David Wolpert and Paul Ampadu. 2012. Temperature effects in semiconductors. In Managing Temperature Effects in Nanoscale Adaptive Systems. Springer, 15-33.

Xilinx. 2011. Spartan-6 Family Overview. Retrieved April 29, 2016 from http://www.xilinx.com/support/ documentation/data_sheets/ds160.pdf. 
Dai Yamamoto, Kazuo Sakiyama, Mitsugu Iwamoto, Kazuo Ohta, Takao Ochiai, Masahiko Takenaka, and Kouichi Itoh. 2011. Uniqueness enhancement of PUF responses based on the locations of random outputting RS latches. In Proceedings of the 13th International Workshop on Cryptographic Hardware and Embedded Systems (CHES'11), Bart Preneel and Tsuyoshi Takagi (Eds.). Springer, Berlin, 390-406. DOI : http://dx.doi.org/10.1007/978-3-642-23951-9_26

Haile Yu, Philip H. W. Leong, and Qiang Xu. 2012. An FPGA chip identification generator using configurable ring oscillators. IEEE Trans. VLSI. Syst. 20, 12 (Dec. 2012), 2198-2207. DOI : http://dx.doi.org/10.1109/ TVLSI.2011.2173770

Received June 2016; revised December 2016; accepted January 2017 